\title{
Otolaryngological Manifestations in HIV Infected Patients, Tehran, Iran
}

Sirous Jafari ${ }^{1}$, Ebrahim Razmpa ${ }^{2}$, Zaker Saeedinejad ${ }^{3}$, Mosa Sadrhosseini' ${ }^{2}$, Koosha Paydary ${ }^{3,5}$, Behtash Saedi ${ }^{2}$, Gholamreza Esmaeeli Djavid ${ }^{3}$, SeyedAhmad SeyedAlinaghi ${ }^{3^{*}}$, Maryam Foroughi ${ }^{3}$ and May Sudhinaraset ${ }^{4}$

${ }^{1}$ Department of Infectious and Tropical Diseases, Tehran University of Medical Sciences, Tehran, Iran

${ }^{2}$ Department of Otolaryngology, Tehran University of Medical Sciences, Tehran, Iran

${ }^{3}$ Iranian Research Center for HIVIAIDS (IRCHA), Tehran University of Medical Sciences, Tehran, Iran

${ }^{4}$ Health Systems Initiative, Global Health Group, University of California, USA

${ }^{5}$ Students Scientific Research Center (SSRC), Tehran University of Medical Sciences, Tehran, Iran

\begin{abstract}
Background: Extant literature finds that $80 \%$ of HIV-infected patients present with otorhinolaryngological symptoms during the course of the infection. To date, no studies have defined HIV-associated ENT manifestations in Iran. To fill this gap in the literature, this study characterizes ENT signs and symptoms in HIV-infected individuals in Tehran, Iran.

Methods: The cross-sectional study consisted of $98 \mathrm{HIV}$ infected patients who were referred to a Voluntary Counseling and Testing (VCT) center of Imam Khomeini hospital, Tehran from September 2007 to September 2008. A detailed history and physical examination was conducted for each patient. A questionnaire based on ENT manifestations was designed for the study. Para clinical assessments were carried out if any ENT manifestation was present. Analysis included calculations of mean values of quantitative associations between ENT symptoms and otological and nasopharyngeal complaints as well as ENT symptoms and demographic characteristics. Statistical analysis was estimated using either Chi-square test or $t$ - test.

Results: Among ENT manifestations, otological and nasopharyngeal complaints were most common. Hearing loss $(61 \%)$, post-nasal discharge $(23.5 \%)$, xerostomia $(39.8 \%)$ and voice change $(23.5 \%)$ were the most frequently cited complaints by participants. In clinical examination, external otitis $(6 \%)$, mucosal dryness of nasopharynx $(6.1 \%)$, candidiasis $(9.2 \%)$, posterior $(7.1 \%)$ and anterior $(6.1 \%)$ lymphadenopathies were also common findings. Lower CD4 count was associated with increased prevalence of oropharyngeal conditions. In addition, the duration of HIV infection was significantly associated with oropharyngeal conditions.
\end{abstract}

Conclusion: ENT evaluations are highly recommended for early diagnosis, especially in patients with specific symptoms in unusual locations.

\section{Keywords: ENT manifestations; HIV infection}

\section{Introduction}

The link between ear, nose and throat (ENT) disease and human immunodeficiency virus (HIV) has been well-established. Studies have found that over $80 \%$ of HIV-infected individuals present with otorhinolaryngological symptoms during the course of infection [13]. In early stages of the infection, oral and nasal manifestations are highly common, and typically diagnosed before HIV has been detected [4]. Oral disease, in particular, is the most common, occurring in approximately half of HIV positive patients [5]. Due to high incidence of HIV worldwide, combined with comorbidity associated with ENT symptoms, ENT specialists and otolaryngologists play a crucial role in diagnosis and treatment of HIV infected patients. Early studies demonstrate that ENT symptoms may result in HIV, are associated with progression as well as treatment failure of HIV [6-10]. Therefore, while ENT symptoms are not diagnostic of HIV, it may suggest infection, and ENT specialists should be equipped with knowledge of ENT manifestations associated with HIV [11].

The HIV lymphadenopathy and lymphoid hyperplasia associated with the immunocompromised state may be observed as a consequence of viral dissemination. Although complications of external ear commonly arise from infections such as Pneumocystis or Tuberculosis, sensorineural hearing loss may result from primary involvement of the central nervous system (CNS) or peripheral auditory nerve. Thereby, pathological mechanisms responsible for such manifestations are either related to opportunistic infections or malignancies associated with the immunocompromised state in HIV patients [7-10].
To date, no previous study has been conducted to determine HIVassociated ENT complications in Iran. Therefore, the aim of this study was to identify the prevalence of ENT complications in HIV infected people in Iran. In addition, we wanted to determine the associations between ENT manifestations, demographic characteristics such as age and gender, and therapeutic features including CD4 count and being on highly active antiretroviral therapy (HAART).

\section{Methods}

\section{Study participants}

From September 2007 to September 2008, 98 HIV-infected patients were recruited to participate in a cross-sectional study. Recruitment took place at the Voluntary Counseling and Testing (VCT) center of Imam Khomeini hospital, Tehran. All participants attended an outpatient ENT clinic where they underwent examination by

${ }^{*}$ Corresponding author: SeyedAhmad SeyedAlinaghi, MD, Iranian Research Center for HIVIAIDS (IRCHA), Tehran University of Medical Sciences, Tehran, Iran, Tel: +98(021) 66947984; Fax: +98(021) 66947984; E-mail: s_a_alinaghi@yahoo.com

Received April 04, 2012; Accepted June 28, 2012; Published July 04, 2012

Citation: Jafari S, Razmpa E, Saeedinejad Z, Sadrhosseini M, Paydary K, et al. (2012) Otolaryngological Manifestations in HIV Infected Patients, Tehran, Iran. J AIDS Clinic Res 3:160. doi:10.4172/2155-6113.1000160

Copyright: (c) 2012 Jafari S, et al. This is an open-access article distributed unde the terms of the Creative Commons Attribution License, which permits unrestricted use, distribution, and reproduction in any medium, provided the original author and source are credited. 
Citation: Jafari S, Razmpa E, Saeedinejad Z, Sadrhosseini M, Paydary K, et al. (2012) Otolaryngological Manifestations in HIV Infected Patients, Tehran, Iran. J AIDS Clinic Res 3:160. doi:10.4172/2155-6113.1000160

Page 2 of 4

\begin{tabular}{|l|l|l|l|l|l|}
\hline Categories & Otological & Nasopharyngeal & Oropharyngeal & Laryngeal & Head \& neck \\
\hline $\begin{array}{l}\text { Symptoms and } \\
\text { complaints }\end{array}$ & Hearing loss, pruritus and tinnitus & Post nasal discharge and rhinorhea & $\begin{array}{l}\text { Xerostomia and } \\
\text { Sore throat }\end{array}$ & Voice change & - \\
\hline Clinical findings & External otitis & Mucosal dryness and crusting & Candidiasis and gingivitis & - \\
\hline
\end{tabular}

Table 1: Common ENT symptoms and clinical findings observed among study participants, Tehran, Iran.

\begin{tabular}{|c|c|c|c|c|c|}
\hline ENT manifestations & Demographic/therapeutic features of patients & All $(n=98)$ & With the manifestations & Without the manifestations & ${ }^{*} \mathbf{P}$ value \\
\hline \multirow{2}{*}{ Otological } & \multirow{2}{*}{$\begin{array}{l}\text { Male } \\
\text { Female }\end{array}$} & $78.3 \%$ & $100 \%$ & $72.7 \%$ & \multirow{2}{*}{0.027} \\
\hline & & $21.7 \%$ & $0 \%$ & $27.3 \%$ & \\
\hline \multirow{2}{*}{ Oropharyngeal } & Duration of HIV infection (months) & $31.46 \pm 27$ & $14.32 \pm 22.78$ & $30.62 \pm 32.78$ & 0.032 \\
\hline & CD4 level (cells/ml) & $310.89 \pm 294.9$ & $163.18 \pm 218.6$ & $353.64 \pm 301.42$ & 0.007 \\
\hline \multirow{3}{*}{ Head and neck } & Age & $35.76 \pm 11.2$ & $30.34 \pm 16.27$ & $37.06 \pm 9.27$ & 0.018 \\
\hline & Male & $80.6 \%$ & $57.9 \%$ & $86.1 \%$ & \multirow{2}{*}{0.005} \\
\hline & Female & $19.4 \%$ & $42.1 \%$ & $13.9 \%$ & \\
\hline
\end{tabular}

*Only significant $P$ values are mentioned defined as $p<0.05$

Table 2: Statistically significant associations between individual-level factors and ENT manifestations in three parts (otological, oropharyngeal, head and neck).

otorhinolaryngologists three times a week. Standard western blot test was performed to confirm HIV status.

\section{Measures}

A standard questionnaire regarding ENT manifestations was filled by an ENT specialist after each participant examination. If any ENT manifestations were identified, para clinical assessments (e.g. audiometry, peripheral nervous system (PNS) CT scan) were carried out. Detailed clinical history was taken and physical examinations were conducted. Demographic characteristics and laboratory tests were obtained via patient interview and profile.

\section{Data analysis}

Mean values for quantitative variables (age, $\mathrm{CD} 4$ count and duration of infection) and the frequency of qualitative data (sex, presence or absence of ENT manifestations and taking or not taking HAART) were analyzed. Quantitative data were reported with \pm SD of their mean values and qualitative data were calculated as relative and absolute prevalence rates. $\mathrm{P}$ values less than 0.05 were considered statistically significant. All analyses were performed using SPSS version 12. Statistical analysis was estimated using either Chi-square test or t- test.

\section{Ethical considerations}

Informed written consent was obtained from all participants. This study was approved by the Institutional Review Board (IRB) of Tehran University of Medical Sciences.

\section{Results}

Of the 98 study participants, approximately $81 \%$ were men $(n=79)$. The mean age of participants was $35.76 \pm 11.2$ years. Of these, 41 patients $(41.8 \%)$ had previously been on HAART and the mean duration of diagnosis was $31.46 \pm 27$ months. The mean CD4 count was $310.89 \pm 294.9$ cells $/ \mathrm{ml}$, and in $42.9 \%$ of the patients, CD4 count was less than $200 \mathrm{cells} / \mathrm{ml}$. Clinical and paraclinical findings were assessed in ear, nose and sinuses, mouth and oropharynx, larynx and neck. Of these otorhinolaryngological symptoms, nasal and oral complaints were the most common among the patients. Table 1 shows the most prevalent complaints and clinical findings among patients.

\section{Otological manifestations}

Approximately $61 \%$ of study participants presented at least one otological manifestation. Almost all of these participants had ear complaints including hearing loss, otalgia, otorrhea, external canal pruritus, tinnitus, vertigo and otorrhagia.

Examination of the external auditory canal in 67 patients, showed external otitis (6\%), otomycosis (3\%) and mass (1.5\%) was observed. Among the 43 patients for whom the tympanic membrane examination was assessed, 34 patients (79.1\%) had normal tympanic membranes, and in one study participant, otitis media with effusion (OME), chronic otitis media (COM) and retracted tympanic membrane were found. Audiometry tests were performed for perforated, retracted, OME, COM and otorrhagy. SDS (speech discrimination score) and SRT (speech recognition testing) were performed among 11 participants, and all reported within clinical normal ranges. Additionally, right and left tympanogram typing were requested for these 11 patients. The right tympanogram was found A type in almost all patients $(n=10)$ and $C$ type in one patient. Similarly, almost all patients $(n=9)$ was A type in the left tympanogram, with one patient each resulting in $B$ and $C$ type.

\section{Nose and sinuses}

Eighty-five percent of study participants presented with nasal and sinus symptoms and $75.5 \%$ had nasal and sinus complaints including rhinorrhea, nasal discharge, facial pain, epistaxis, nasal obstruction, post-nasal discharge, anosmia and hyposmia. Discharge was observed in 23 of the patients (23.5\%). Other clinical findings included mucosal dryness (6.1\%), crusting (5.1\%), edema (4.1\%) and septal perforation (1\%). In the case of septal perforation and nasopharynx edema $(n=5)$, PNS CT was performed and rhinosinusitis was found in only one patient. Nasopharyngoscopy for all patients were normal.

\section{Mouth and oropharynx}

A majority of patients tested positive for oral and oropharyngeal clinical manifestations $(\mathrm{n}=62,62.2 \%)$. Patients indicated xerostomia (39.8\%), repeated sore throat (13.3\%), dysphagia (12.2\%), burning tongue $(9.2 \%)$, odynophagia $(8.2 \%)$ and other complaints $(3.1 \%)$. In physical examinations, study participants showed symptoms of gingivitis (11.2\%), candidiasis (9.2\%), aphthous (4.1\%), mass (1\%) and others $(3.1 \%)$.

\section{Larynx}

Larynx associated symptoms occurred in 45 patients (45.9\%). Voice change (23.5\%) and hoarseness (17.3\%) were the most common 
Citation: Jafari S, Razmpa E, Saeedinejad Z, Sadrhosseini M, Paydary K, et al. (2012) Otolaryngological Manifestations in HIV Infected Patients, Tehran, Iran. J AIDS Clinic Res 3:160. doi:10.4172/2155-6113.1000160

manifestations; however, nodules and edema were also reported in $7.5 \%$ of study participants.

\section{Head and neck}

Clinical manifestations of the head and neck were observed in 19 patients (19.4\%). Pigmentation and parotid enlargement were reported in two patients. Fungal infection and solitary mass were observed in one patient. Lymphadenopathy of the head and neck was observed in 14 patients $(14.3 \%)$ including posterior cervical $(7.1 \%)$, anterior cervical (6.1\%), pre auricular $(4.1 \%)$, posterior auricular $(4.1 \%)$, occipital (3.1\%), submental (1\%), and sub mandibular (1\%).

As shown in Table 2, there was a statistically significant difference between men and women in otological manifestations $(\mathrm{p}<0.05)$. Men demonstrated greater otological findings compared to women. Another significant difference was observed between CD4 count and oropharyngeal manifestations. Longer duration of infection was associated with greater oropharyngeal symptoms. In addition, greater frequency of head and neck symptoms was positively associated with age and male gender $(\mathrm{p}<0.05)$.

\section{Discussion}

To our knowledge, this is the first study to characterize ENT signs and symptoms among HIV-infected individuals living in Tehran, Iran. Using a convenience sample from a VCT clinic, we found that the most common otological symptoms were hearing loss and tinnitus. These manifestations were observed among $61.2 \%$ of study participants, which is in line with other studies that have reported a wide range between $11.5 \%-71.4 \%$ among HIV-infected individuals [12-14]. These results are important for the clinical care of HIV-infected patients. Providers should be recognize the high prevalence of ENT symptoms found among this vulnerable population.

External otitis and perforated tympanic membrane were the most prevalent manifestations meanwhile other studies have demonstrated recurrent otitis media [15], COM [16] and AOM [17] as common symptoms among HIV-infected individuals. These data indicate the need to recognize external otitis and perforated tympanic membrane manifestations as additional common symptoms. Surprisingly, underlying sensorineural mechanism was not found to be responsible for hearing loss detected according to audiometry tests performed. Other studies have documented sensorineural defects as a pathological explanation for the common hearing loss observed among HIV infected patients [18]. One possible explanation for this difference is that study participants were in the primary stages of infection and therefore, sensorineural mechanisms were not detected.

Candidiasis is one of the most critical clinical complications among HIV-infected patients. In this study, gingivitis and candidiasis were commonly observed; however, other studies have reported a wide range between $14.6 \%$ to $86 \%$ [19]. The positive association between oropharyngeal frequency and CD4 levels may be due to viral infection progression. There was also a positive association between oropharyngeal manifestations and chronicity of the infection.

Moreover, results indicate that being on HAART was not associated with ENT manifestations, which has produced mixed results in prior literature [20,21]. While some studies have also found no association between being on HAART and ENT manifestations [20], other studies have found that HAART may cause a considerable decline in incidence rate of ENT manifestations due to elevated levels of CD4 count [21].

This study found that age was associated with otological complications among HIV-infected individuals. For example, recurrent otitis media was frequently observed among HIV-infected pediatric patients. One hypothesis for this finding is that children experience depressed cellular immunity and eustachian tube dysfunction [22]. In addition, posterior and anterior cervical lymphadenopathies were highly common symptoms found among this population, and that younger individuals were more likely to experience these manifestations compared elderly patients $(\mathrm{p}<0.05)$.

Finally, this study found that gender was associated with presenting with otological symptoms. Compared to women, men are more likely to present with a number of otological manifestations [23]. Specifically, this study found that men were more likely to report nasopharynx complications, particularly due to post-nasal discharge. The study found that men were more likely to report head and neck symptoms compared to women $(\mathrm{p}<0.05)$, which has been validated by other studies [24].

As with any study, there are limitations to our findings. First, due to the sample recruitment within clinics, these results may be an underestimation of prevalence of ENT manifestations. Due to high social stigma of HIV/AIDS in Iran, individuals may be less inclined to seek out HIV/AIDS diagnosis. Second, the small sample size is also a limitation of the study. Stratified results of age and gender may not be powered to produce valid results. Lastly, due to the cross-sectional nature of the study, causality between ENT and HIV infection could not be assessed. Incidence of ENT manifestations would best be determined using a longitudinal study design.

\section{Conclusion}

In conclusion, ENT evaluations by clinicians among HIV infected patients are highly recommended. HIV replication in CD4 positive cells renders the body more susceptible to opportunistic infections and neoplastic disorders. Such pathogens spread quickly in the vulnerable host, resulting in the emergence of uncommon symptoms and malignancies reported in immunocompromised patients [25-27]. Therefore, further investigations should be required in all complicated cases, particularly consultations with ENT specialists given the high prevalence of ENT manifestations. It is particularly crucial to diagnosis these symptoms early to ensure prompt treatment [28]. Early diagnosis of HIV infection via recognition of manifestations ensures longer survival of patients.

It is particularly important to note that ENT conditions may be occur in both HIV-positive and negative patients; however, specific symptoms are only reported in unusual locations and in a more aggressive fashion among HIV-positive infected individuals. Recognition of localized manifestations of the head and neck may improve the clinician's ability to diagnose HIV infection clinically and provide the patients with the best chances for timely and effective treatment.

\section{References}

1. Prasad HK, Bhojwani KM, Shenoy V, Prasad SC (2006) HIV manifestations in otolaryngology. Am J Otolaryngol 27: 179-185

2. Moazzez AH, Alvi A (1998) Head and neck manifestations of AIDS in adults. Am Fam Physician 57: 1813-1822.

3. Baccaglini L, Atkinson JC, Patton LL, Glick M, Ficarra G, et al. (2007) Management of oral lesions in HIV-positive patients. Oral Surg Oral Med Oral Pathol Oral Radiol Endod 103: S50.e1-23.

4. Campanini A, Marani M, Mastroianni A, Cancellieri C, Vicini C (2005) Human immunodeficiency virus infection: personal experience in changes in head and neck manifestations due to recent antiretroviral therapies. Acta Otorhinolaryngol Ital 25: 30-35. 
Citation: Jafari S, Razmpa E, Saeedinejad Z, Sadrhosseini M, Paydary K, et al. (2012) Otolaryngological Manifestations in HIV Infected Patients, Tehran, Iran. J AIDS Clinic Res 3:160. doi:10.4172/2155-6113.1000160

5. Johnson NW, Glick M, Mbuguye TN (2006) Oral health and general health. Adv Dent Res19: 118-121.

6. Truitt TO, Tami TA (1999) Otolaryngologic manifestations of human immunodeficiency virus infection. Med Clin North Am 83: 303-315.

7. Tami T, Gold SM (1992) Disease of the nose and paranasal sinuses in the human immunodeficiency virus-infected population. Otolaryngol Clin North Am 25: $1199-1210$

8. Lalwani AK, Sooy CD (1992) Otologic and neurotologic manifestations of acquired immunodeficiency syndrome. Otolaryngol Clin North Am 25: 11831197.

9. Rarey KE (1990) Otologic pathophysiology in patients with human immunodeficiency virus. Am J Otolaryngol 11: 366-369.

10. Soucek S, Michaels L (1996) The ear in the acquired immunodeficiency syndrome: clinical and audiologic investigation. Am J Otol 17: 35-39.

11. lacovou E, Vlastarakos PV, Papacharalampous G, Kampessis G, Nikolopoulos TP (2012) Diagnosis and treatment of HIV-associated manifestations in otolaryngology. Infect Dis Rep 4: 22-29.

12. Ndjolo A, Njock R, Ngowe NM, Ebogo MM, Toukam M, et al. (2004) Early ENT manifestations of HIV infection/AIDS. An analysis of 76 cases observed in Africa. Rev Laryngol Otol Rhinol (Board) 125: 39-43.

13. Marcusen DC, Sooy CD (1985) Otolaryngologic and head and neck manifestations of acquired immunodeficiency syndrome (AIDS). Laryngoscope 95: 401-405.

14. Sant'Anna GD, Franche GL, Tabajara LM, Favero RM, Kuhl G (1991) Otorhinolaryngologic manifestations of acquired immunodeficiency syndrome. Rev Laryngol Otol Rhinol (Bord) 112: 63-66.

15. Chen AY, Ohlms LA, Stewart MG, Kline MW (1996) Otolaryngologic disease progression in children with human immunodeficiency virus infection. Arch Otolaryngol Head Neck Surg 122: 1360-1363.

16. Bernaldez PC, Morales G, Hernandez CM (2005) Chronic suppurative otitis media in HIV-infected children: P140. Otolaryngol Head Neck Surg 133: 243244.
17. Chandrasekhar SS, Connelly PE, Brambhatt SS, Shah CS, Kloser PC, et al. (2000) Otologic and audiologic evaluation of human immunodeficiency virusinfected patients. Am J Otolaryngol 21: 1-9.

18. Mata Castro N, Yebra Bango M, Tutor de Ureta P, Villarreal Garcia-Lomas M, Garcia Lopez F (2000) Hearing loss and human immunodeficiency virus infection. Study of 30 patients. Rev clin Esp 200: 271-274.

19. Herdman RC, Forster S, Stafford ND, Pinching AJ (1989) The recognition and management of the otolaryngological manifestations of AIDS. Clin Otolaryngol Allied Sci 14: 323-332.

20. Zuccotti GV, D’Auria E, Torcoletti M, Lodi F, Bernardo L, et al. (2001) Clinica and pro-host effects of cefaclor in prophylaxis of recurrent otitis media in HIVinfected children. J Int Med Res 29: 349-354.

21. Colebunders R, Francis H, Mann JM, Bila KM, Kandi K, et al. (1988) Parotid Swelling During Human Immunodeficiency Virus Infection. Arch Otolaryngol Head Neck Surg 114: 330-332.

22. Church J (1987) HIV infection at children's hospital Los Angeles: recurrent otitis media or chronic sinusitis at the presenting process in pediatric AIDS. Immunol Allergy 9: 25-32.

23. Ondzotto G, Ibara JR, Mowondabeka P, Galiba J (2004) Cervico-facial and ENT symptoms due to HIV infection in tropical area. About 253 Congolese cases. Bull Soc Pathol Exot 97: 59-63.

24. Deb T, Singh NB, Devi HP, Sanasam JC (2003) Head and neck manifestations of HIV infection: a preliminary study. J Indian Med Assoc 101: 93-95.

25. Lucente FE (1991) Otolaryngologic aspects of acquired immunodeficiency syndrome. Med Clin North Am 75: 1389-1398.

26. Youngs $R$ (1997) Human immunodeficiency virus in otolaryngology. J Laryngo Otol 111: 209-211.

27. Corey JP, Seligman I (1991) Otolaryngology problems in the immune compromised patient--an evolving natural history. Otolaryngol Head Neck Surg 104: 196-203.

28. Birchall MA, Horner PD, Stafford ND (1994) Changing patterns of HIV infection in otolaryngology. Clin otolaryngol Allied Sci 19: 473-477. 